\section{BACE failures lower AD expectations, again}

There's more bad news for $\beta$-secretase 1 (BACE1) inhibitors, a class of Alzheimer disease (AD) drug designed to reduce amyloid plaques by controlling amyloid processing upstream of anti-amyloid antibodies and of $\gamma$-secretase inhibitors. Just weeks after Merck \& Co. published the results of its failed pivotal trial of verubecestat in mild-to-moderate $A D$, Johnson $\&$ Johnson and partner Shionogi have terminated late-stage development of atabecestat in asymptomatic individuals owing to safety concerns.

Merck flagged up the problems with its verubecestat last year, terminating a trial in mild-to-moderate disease due to futility. Full trial results in the New England Journal of Medicine now show that two different doses of drug did not slow the clinical progression of mild-to-moderate disease, despite achieving near-maximal reduction of amyloid- $\beta$ (A $\beta$ ) in cerebrospinal fluid and a modest reduction in brain amyloid load. "This suggests that once dementia is present, disease progression may be independent of $A \beta$ production or, alternatively, that the amyloid hypothesis of Alzheimer's disease may not be correct," the authors conclude.

Even after this failure in mild-to-moderate disease, Merck continued running a phase III trial of verubecestat in patients with prodromal $A D$, who have a history of memory decline and positive amyloid imaging but the absence of full-blown dementia. The company suspended that trial in February, however, on the basis of an interim analysis of the overall benefit-risk profile of the drug.

Johnson \& Johnson and partner Shionogi were testing their atabecestat earlier still in the disease process, in asymptomatic patients at risk of developing disease. They have now halted this study after liver enzyme levels spiked in some participants.

Several drug developers nevertheless remain committed. Novartis and partner Amgen are testing their BACE inhibitor CNP520 in a phase II/III trial in asymptomatic individuals at risk of developing disease. Eisai and partner Biogen, as well as AstraZeneca and partner Eli Lilly, have phase III trials of BACE inhibitors ongoing in subjects with early AD.

Drug developers are also starting to venture beyond amyloid-centric approaches to AD drug development. Denali Therapeutics recently advanced its RIPK1 inhibitor into clinical trials, highlighting renewed interest in neuroinflammatory strategies and microglia-modulating drugs.

Asher Mullard

\section{On the origin of transformative drugs}

In the latest attempt to unpick the relative benefit of basic versus applied research, researchers have charted the drug discovery and development timelines of 28 'transformative' drugs. Nearly $80 \%$ of these medicines could be traced back to one or several basic science discoveries, they report in $\underline{\text { Science Translational Medicine. }}$.

The average time from first basic discovery to FDA approval was 31 years, with no differences for small molecules versus biologics. There was a trend towards shorter timelines for newer medicines, with a 26 year lag between seminal discovery and approval.

The analysis was run by Mark Fishman, former president of Novartis Institutes for BioMedical Research (NIBR) who is now at Harvard University, and his industry colleagues. The 28 'transformative drugs' were selected on the basis of an article published in 2013 in Nature Reviews Drug Discovery that surveyed more than 180 physicians across all medical areas to identify a short-list of important medicines. Fishman and his colleagues then worked with 80 experts to define the discoveries that led to those 28 medicines.

"For the vast majority of the medicines we examined, the foundational discoveries were made by scientists trying to understand nature. They had no evident intent to make a drug. In fact, for most of these drugs, decades elapsed and insights accumulated to refine the original observation. Only then did the potential for a new drug become clear and the search was on for a chemical or protein that might become a therapy," the authors wrote in an associated opinion piece in STAT.

"Judging from the past, the development of new drugs will continue to be a lengthy journey. The first step, often recognized only in retrospect, likely will be an insight in yeast or fish or mice without any obvious relevance to a new medical breakthrough. There's really no shortcut. The only way to lay the foundation for the next generation of new medicines is to invest in learning the fundamental truths about how the body functions and how it falls apart," they conclude.

The paper's supplementary information includes detailed discovery and development timelines for all 28 transformative medicines.

Asher Mullard

\section{FDA approves first-in-class SYK inhibitor}

The FDA approved Rigel Pharmaceuticals' first-in-class SYK inhibitor fostamatinib for the treatment of adult patients with chronic immune thrombocytopenia (ITP) who have had an insufficient response to a previous treatment.

ITP is a rare disease in which the immune system attacks and destroys the body's own blood platelets. Current treatment options for ITP include steroids, splenectomy, thrombopoietin receptor agonists and more. But not all patients have an adequate response to these existing therapies.

SYK has a key role in the adaptive immune system, mediating immunoreceptor signalling in macrophages, neutrophils, mast cells and $B$ cells. In ITP, inhibition of SYK with fostamatinib reduced antibody-mediated destruction of platelets in clinical trials, leading to platelet stabilization in 16-18\% of patients, compared with $0-4 \%$ of patients receiving placebo. Serious adverse drug reactions included febrile neutropenia, diarrhoea, pneumonia and hypertensive crisis.

Drug developers once had bigger plans for this drug class. Rigel licensed the drug to AstraZeneca in 2010 for development and commercialization in rheumatoid arthritis and haematological malignancies, potentially much more profitable indications. AstraZeneca advanced it into phase III rheumatoid arthritis trials that same year, but then discontinued all development in 2013 and returned rights to Rigel "due to safety and efficacy reasons". Investigators recently revisited these failed trials, and found that translational biomarker assessments may have been able to predict the results ahead of time.

A few other companies, including Gilead Sciences and Takeda Pharmaceuticals, are still persevering with SYK inhibitors in phase I/II oncology trials.

Asher Mullard 